\title{
LGR6 promotes the progression of gastric cancer through PI3K/AKT/mTOR pathway
}

This article was published in the following Dove Press journal:

OncoTargets and Therapy

\section{Jing $\mathrm{Ke}^{1,2}$ \\ Peng $\mathrm{Ma}^{2}$ \\ Jinpeng Chen ${ }^{2}$ \\ Jun Qin $^{2}$ \\ Haixin Qian'}

'Department of General Surgery, The First Affiliated Hospital of Soochow University, Suzhou, People's Republic of China; ${ }^{2}$ Department of General Surgery, The Affiliated Hospital of Nantong University, Nantong, People's Republic of China
Correspondence: Haixin Qian Department of General Surgery, The First Affiliated Hospital of Soochow University, No. 188 Shi Zi Street, Suzhou 215006, People's Republic of China Tel +86051265223637

Email qhxsoochow@I63.com
Background: In the present study, we aimed to investigate the role of LGR6 in the progression of gastric cancer (GC) and explore the intrinsic molecular mechanisms.

Materials and methods: The lentiviral LGR6 shRNA (sh-LGR6) and lentiviral expression vector of LGR6 gene (OE-LGR6) were used to regulate the LGR6 expression. Furthermore, we performed in vitro experiments to observe whether PI3K/AKT/mTOR pathway was affected by LGR6 and assess the role of LGR6 in the proliferation, apoptosis, migration, and invasion of GC cells.

Results: Our data showed that phosphorylated AKT and mTOR were downregulated by sh-LGR6 $(P<0.05)$. The expressions of proapoptotic proteins Bax and Caspase-3 were upregulated by sh-LGR6 $(P<0.05)$; the expression of antiapoptotic protein $\mathrm{Bcl} 2$ was downregulated by sh-LGR6 $(P<0.001)$. Besides, the functional experiments proved that sh-LGR6 could promote the apoptosis of GC cells and inhibit the proliferation, invasion, and migration of GC cells $(P<0.001)$. Compared with sh-LGR6, OE-LGR6 led to the opposite results.

Conclusion: LGR6 is an antiapoptosis protein which controls the progression of GC through $\mathrm{PI} 3 \mathrm{~K} / \mathrm{AKT} / \mathrm{mTOR}$ pathway. More in vivo experiments and clinical trials are necessary to confirm the possibility of LGR6 in tumor therapy.

Keywords: LGR6, pathway, tumor therapy, gastric cancer

\section{Introduction}

Gastric cancer (GC) is one of the important diseases threatening human health in the world, and it is the second cause of cancer-related deaths in women and the third in men in China. ${ }^{1,2}$ At present, surgical resection is still the main treatment for GC. However, the resection rate for GC was only about $50 \%$ because most of patients with GC are diagnosed at advanced stage. ${ }^{3}$ Chemotherapy can only alleviate the symptoms and prolong the survival of patients with GC, and there is still a lack of "wonder drugs" for GC. ${ }^{4}$ To improve the prognosis of GC, early diagnosis is of utmost importance. ${ }^{5}$ Moreover, more effective therapeutic strategies still need to be instituted, which depend on new therapeutic targets. Investigating the molecular mechanisms of GC progression is crucial to identify new therapeutic targets.

LGR6 is a member of the leucine-rich repeat containing G-protein-coupled receptors (LGRs). ${ }^{6}$ In some organs, such as skin, taste buds, and lungs, LGR6 has been identified as a stem cell marker. ${ }^{7-9} \mathrm{LGR}^{+}$stem cells play an important role in hair follicle development and wound repair. ${ }^{6,10}$ In addition, LGR6 is related to the prognosis of patients with tumor. Guinot et $\mathrm{al}^{11}$ found that LGR6 ${ }^{+}$cells contributed to human lung adenocarcinomas. The lung stem cell marker LGR6 becomes enriched from early to late stages in non-small-cell lung cancer cells. ${ }^{11} \mathrm{Krejs}^{12}$ reported that LGR6 is overexpressed in GC and patients with high LGR6 expression had poor survival. 
They suggested that LGR6 could promote the progression of tumor through Wnt pathway. LGR6, and its homologous receptors, are amplifiers of Wnt pathway and involved in maintaining stem cell self-renewal. ${ }^{13} \mathrm{Wnt}$ pathway plays an important role in the process of cell differentiation, proliferation, and apoptosis, as well as in the cell carcinogenesis and tumor invasion. ${ }^{14}$

Considering the complex interaction of signaling pathways, we hypothesized that there are some other pathways involved in the tumor progression regulated by LGR6. The present study is proposed to identify the new pathway controlled by LGR6 and explore the effects of LGR6 on the proliferation, apoptosis, migration, and invasion of GC cells in vitro, thus contributing to further understand the molecular mechanisms of GC progression and develop more effective therapeutic strategies.

\section{Materials and methods}

\section{Cell culture and reagents}

Human GC cell lines (MGC803 and MKN45) were obtained from the Shanghai Institutes of Biological Sciences Cell Bank and were maintained in Roswell Park Memorial Institute-1640 (RPMI 1640) supplemented with 10\% fetal bovine serum (Gibco, Thermo Fisher Scientific, Waltham, MA, USA) and 1\% penicillin/streptomycin (Gibco, Thermo Fisher Scientific) at $37^{\circ} \mathrm{C}$ in a humidified atmosphere containing $5 \% \mathrm{CO}_{2}$. Antibodies against Caspase-3, $\mathrm{Bcl} 2, \mathrm{Bax}$, $\mathrm{AKT}, \mathrm{mTOR}$, and $\beta$-actin as well as phosphorylated forms of AKT (T308) and mTOR (S2448) were purchased from Abcam (Cambridge, UK). Bound antibodies were detected with horseradish peroxidase-linked antibody against mouse (Abcam) or rabbit (Santa Cruz Technology, Dallas, TX, USA) immunoglobulin G, followed by enhanced chemiluminescence detection (Amersham, USA).

\section{Gene transduction}

The shRNAs targeting LGR6 (LGR6 shRNA1, 2, 3), lentiviral expression vector of LGR6 gene (OE-LGR6), and their negative control were obtained from Ribobio (Guangzhou, People's Republic of China) (Table 1). The lentivirus was packaged using the pSPAX2, pMD2G, and pHBLV-U6ZsGreen-Puro plasmids, as well as Lipofectamine 2000 (Invitrogen, Carlsbad, CA, USA; Thermo Fisher Scientific) for the $293 \mathrm{~T}$ cell line; the lentivirus was collected after 48 h. The MGC803 and MKN45 cell lines were infected with lentivirus and polybrene (1:500; Sigma-Aldrich Co., St. Louis, MO, USA). The expression change of LGR6 was determined by reverse-transcription polymerase chain reaction (RT-PCR) at $48 \mathrm{~h}$ after transfection.
Table I The sequence of LGR6 shRNAs

\begin{tabular}{ll}
\hline Group & Sequences $\left(\mathbf{5}^{\prime}\right.$ to $\left.\mathbf{3}^{\prime}\right)$ \\
\hline LGR6 shRNAI & CCCUGGAUCUUAGCUGGAATT \\
UUCCAGCUAAGAUCCAGGGTT \\
LGR6 shRNA2 & GCAUUCCAGUACCUGCCUATT \\
& UAGGCAGGUACUGGAAUGCTT \\
LGR6 shRNA3 & CCUGGAACUGUCUCACAAUTT \\
Negative control & AUUGUGAGACAGUUCCAGGTT \\
& UUCUCCGAACGUGUCACGUTT \\
& ACGUGACACGUUCGGAGAATT \\
\hline
\end{tabular}

\section{RT-PCR}

Total RNA was isolated from GC cells using the Trizol reagent (Takara Bio Inc., Kusatsu, Shiga Prefecture, Japan). Purified RNA was reversely transcribed into cDNA with the M-MLV First Strand kit (Invitrogen) according to the manufacturer's instructions. ${ }^{15}$ RT-PCR reactions were carried out using the SYBR Premix Ex Tag II (Takara Bio Inc.) on an ABI PRISM 7500 Sequence Detection system (Applied Biosystems, Foster City, CA, USA). ${ }^{16}$ Briefly, after an initial denaturation step at $95^{\circ} \mathrm{C}$ for $30 \mathrm{~s}$, amplifications were conducted with 40 cycles at a melting temperature of $95^{\circ} \mathrm{C}$ for $5 \mathrm{~s}$, and an annealing temperature of $60^{\circ} \mathrm{C}$ for $34 \mathrm{~s}$. Human GAPDH was used as the housekeeping gene to normalize the expression level of target gene. Primers used for amplifications were as follows:

Briefly, after an initial denaturation step at $50^{\circ} \mathrm{C}$ for $2 \mathrm{~min}$ and $95^{\circ} \mathrm{C}$ for $2 \mathrm{~min}$, amplifications were conducted with 40 cycles at a melting temperature of $95^{\circ} \mathrm{C}$ for $15 \mathrm{~s}$, and an annealing temperature of $60^{\circ} \mathrm{C}$ for $15 \mathrm{~s}, 72^{\circ} \mathrm{C}$ for 1 min. Human GAPDH was used as the housekeeping gene to normalize the expression level of target gene. Primers used for amplifications were as follows: LGR6 forward, 5'-TGACGGCTTACCTGGACCTCA-3', reverse, 5'-AGAGAATGCTTGTCCTGGGATG-3'; Bcl-2, forward, 5'-CAGGAAACGGCCCGGAT-3', reverse 5'-CTGGGGCCTTTCATCCTCC-3'; Bax forward, 5'-GGGTTGTCGCCCTTTTCTAC-3'; reverse, 5'-CTGGAG ACAGGGACATCAGT-3'; Caspase-3, forward, 5'-TGCT ATTGTGAGGCGGTTGTAG-3', reverse, 5'-GGCACAC CCACCGAAAAC-3'; GAPDH, forward, 5'-GAAGGTG AAGGTCGGAGTC-3'; reverse, 5'-GAAGATGGTGATG GGATTTC-3'.

\section{Western blotting analysis}

Cell lysates were centrifuged at $4^{\circ} \mathrm{C}$ for $10 \mathrm{~min}$ at $12,000 \mathrm{~g}$. Equal amounts of proteins were loaded onto $8 \%$ or $10 \%$ sodium dodecyl sulfate-polyacrylamide gels and then transferred onto polyvinylidene fluoride membranes (Millipore, 
Billerica, MA, USA). After blocking with 5\% BSA/P-BST buffer for $1 \mathrm{~h}$, membranes were incubated in primary antibodies at $4{ }^{\circ} \mathrm{C}$ overnight. Subsequently, blots were incubated with horseradish peroxidase-linked secondary antibody at room temperature for $2-3 \mathrm{~h}$. Immunoreactive proteins were detected using the SuperSignal West Pico Chemiluminescent substrate (Thermo Fisher Scientific). The band quantification was conducted using ImageJ (National Institutes of Health, Bethesda, MA, USA). ${ }^{17}$

\section{Proliferation assays}

MTT assay was used to measure the cell viability of MGC803 and MKN45 cells. $100 \mu \mathrm{L}$ GC cell suspension containing 2,000 cells were seeded in one well in a 96-well plates and incubated for $24,48,72$, and $96 \mathrm{~h}$ at $5 \% \mathrm{CO}_{2}$, at $37^{\circ} \mathrm{C}$. Then, $100 \mu \mathrm{L}$ of MTT solution was added to each well and incubated for $4 \mathrm{~h}$ at $5 \% \mathrm{CO}_{2}$, at $37^{\circ} \mathrm{C}$. Then $100 \mu \mathrm{L}$ DMSO was added to each well and incubated again for $4 \mathrm{~h}$ at $5 \%$ $\mathrm{CO}_{2}$, at $37^{\circ} \mathrm{C}$, until the dissolution of all the formazan; then, absorbance was measured at $490 \mathrm{~nm}$ using Multiscan Plate Reader (Thermo Fisher Scientific, Loughborough, UK). The activity curve was then created. Each process was repeated three times.

\section{Cell apoptosis assay}

Apoptosis of MGC803 and MKN45 cells was evaluated by flow cytometry using the Annexin V PE Apoptosis kit (BD Biosciences, San Jose, CA, USA).$^{18}$ Cells were washed with cold PBS and resuspended in $100 \mu \mathrm{L}$ binding buffer (1×), followed by addition of $5 \mu \mathrm{L}$ Annexin V-PE and $5 \mu \mathrm{L}$ 7-AAD. The cells were incubated for $10 \mathrm{~min}$ at room temperature in the dark. Finally, $400 \mu \mathrm{L}$ binding buffer were added to the cells, which were analyzed by flow cytometry.

\section{Transwell assays}

Cell migration was measured using cell culture inserts (24-well type, $8 \mu \mathrm{m}$ pore size; Corning Inc., Corning, NY, USA). Subsequently, $1 \times 10^{5} \mathrm{GC}$ cells were added into the upper chambers with the serum-free RPMI 1640 medium, while the lower chambers were filled with $500 \mu \mathrm{L}$ complete RPMI 1640. After $16 \mathrm{~h}$ of incubation, the cells that had migrated to the undersurface of the membrane were fixed in $100 \%$ methanol and stained with crystal violet. Cells were visualized and 10 random fields were counted under a microscope.

\section{Wound healing assay}

Cells were plated in six-well plates. After the cells reached $80 \%$ confluence, scratches were made using a $100 \mu \mathrm{L}$ pipette tip. Wound healing was observed and the migration distance was imaged at different time points.

\section{Statistical analysis}

Statistical analysis was performed using SPSS v21.0 (SPSS, Chicago, IL, USA) and GraphPad Prism v5.0 (GraphPad Software, La Jolla, CA, USA) software. Difference between two groups was assessed using Student's $t$-test. Data are presented as the mean \pm standard error of the mean. All experiments, consisting of three replicates, were performed at least twice independently. A $P$-value $<0.05$ was considered as statistically significant, unless otherwise stated.

\section{Result \\ LGR6 influences the protein and mRNA expression of gastric cells}

To determine whether LGR6 plays an important role in the protein and mRNA expression in gastric cells, we first performed shRNA-mediated gene silencing of LGR6 in human GC cell lines MGC803 and MKN45 through lentiviral genes with three different shRNA constructs. The efficiency of gene silencing was confirmed by RTPCR (Figure 1). Then, we chose the most efficient shRNA construct (shRNA3) to transfect cells ( $P<0.001$, Figure 1). By comparing control group with sh-LGR6 group, we verified the role of downregulation of LGR6. Moreover, we compared sh-LGR6 group with sh-LGR6+OE-LGR6 group to further confirm the regulatory effect of overexpression of LGR6.

The RT-PCR analysis showed that sh-LGR6 could suppress the mRNA expression of $\mathrm{Bcl} 2$, and upregulate the mRNA expressions of Caspase 3 and $\mathrm{Bax}(P<0.05$; Figure 2); OR-LGR6 could counterregulate these mRNA expressions $(P<0.05$; Figure 2$)$. Besides, Western blot was used to detect the effects of sh-LGR6 and OE-LGR6 on the protein level of GC cells. The result showed the effects of sh-LGR6 and OE-LGR6 on the protein expressions of Bcl2, Caspase 3, and Bax were consistent with that on mRNA expressions (Figure 3).

\section{LGR6 regulates the PI3K/Akt/mTOR signaling pathway}

The results of Western blot showed that the expression of phosphorylated AKT and mTOR were significantly downregulated by sh-LGR6 ( $P<0.05$; Figure 3); OE-LGR6 could upregulate the expression of phosphorylated AKT and mTOR $(P<0.05$; Figure 3$)$. The results indicated that downregulation of LGR6 inhibited the activation of 

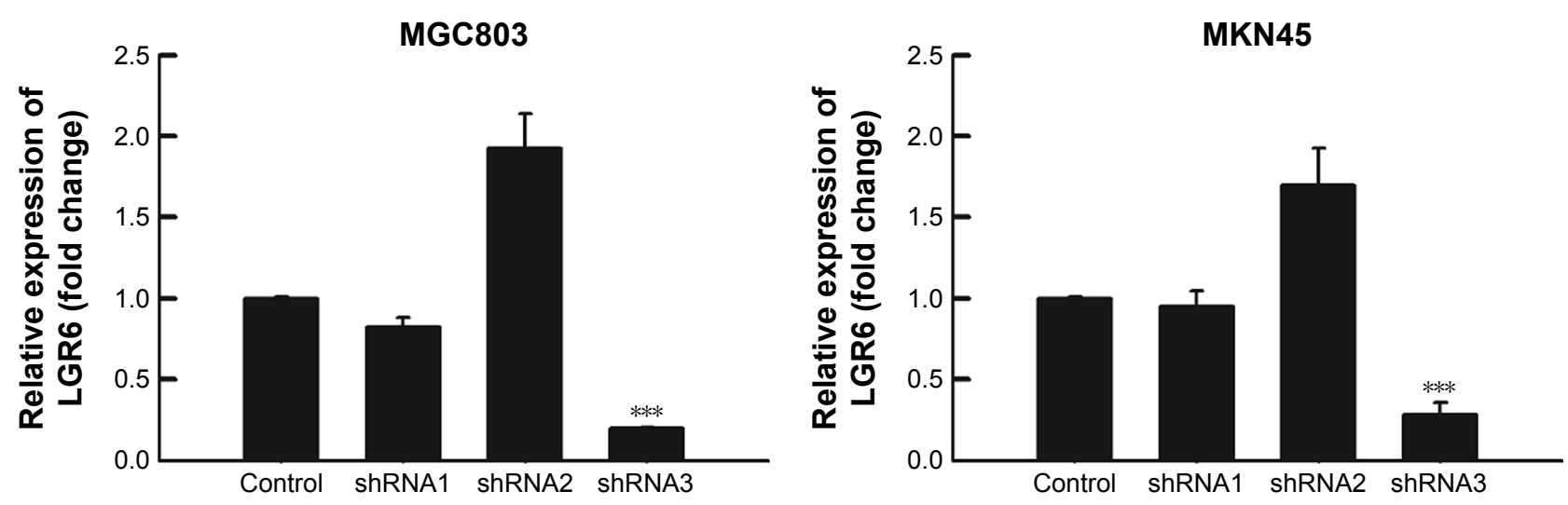

Figure I The gene silencing efficiencies of three shRNA constructs.

Notes: Cell lines (MGC803 and MKN45) were treated with control and shRNAI, 2, and 3 for $48 \mathrm{~h}$. LGR6 expression at the mRNA level was determined by RT-PCR and expressed as fold change relative to the vehicle group. Each column is shown as the mean of three separate experiments; bars, $S D$. $* * * P<0.001$.

Abbreviations: RT-PCR, reverse-transcription polymerase chain reaction; SD, standard deviation.
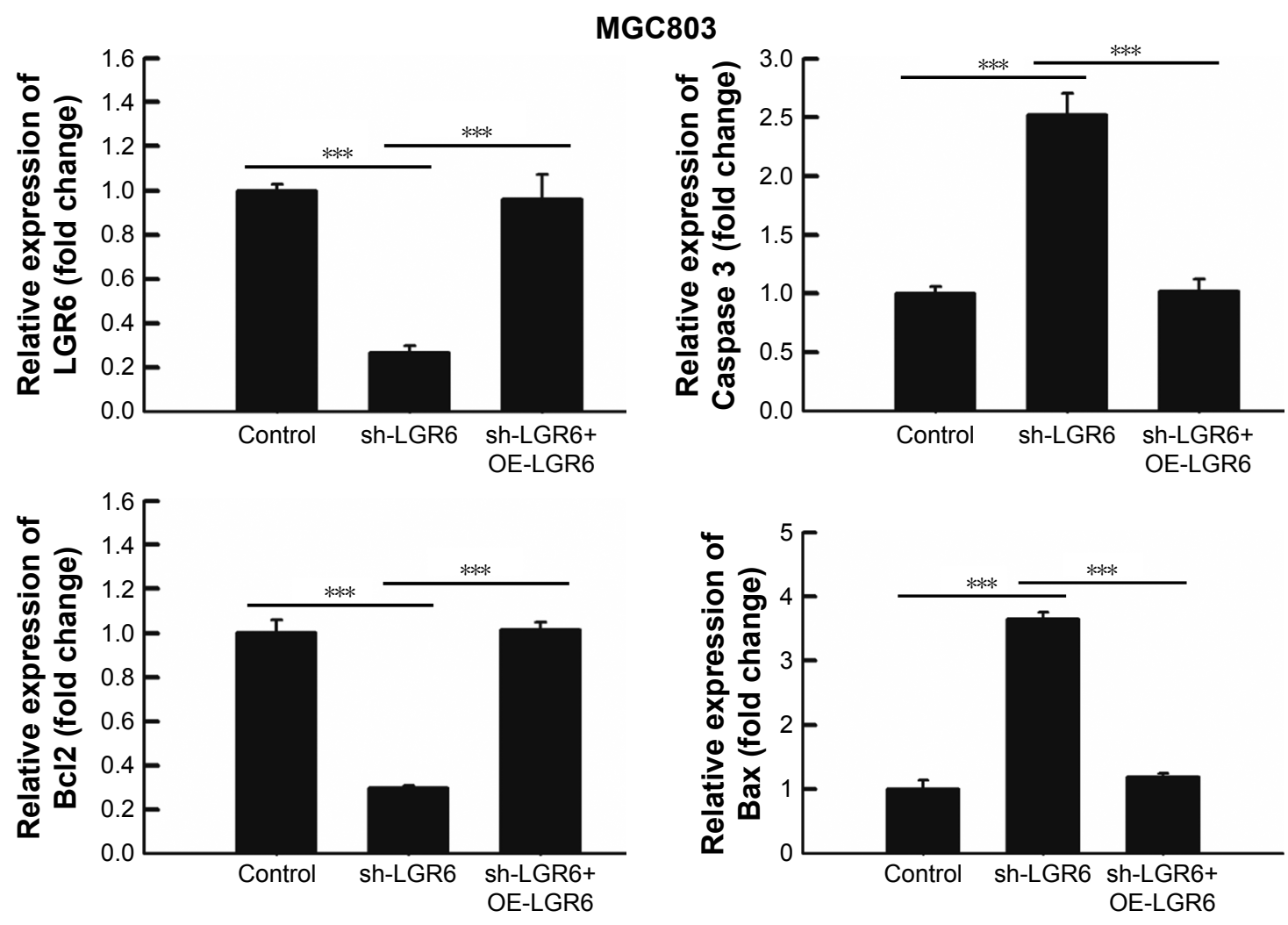

MKN45
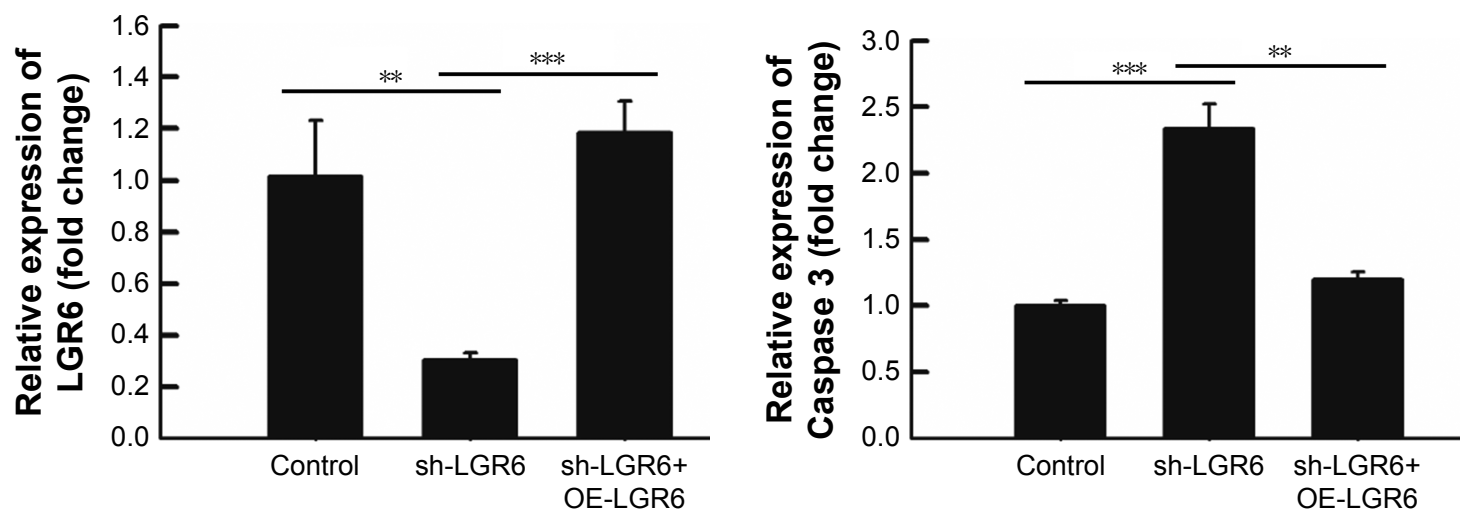

Figure 2 (Continued) 


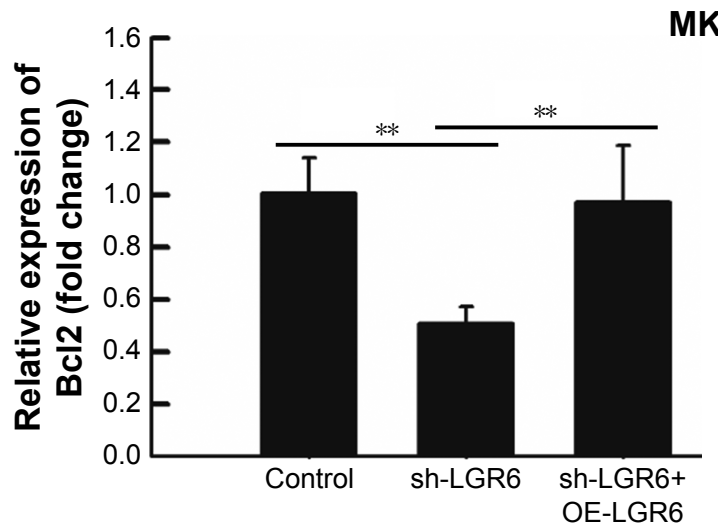

MKN45

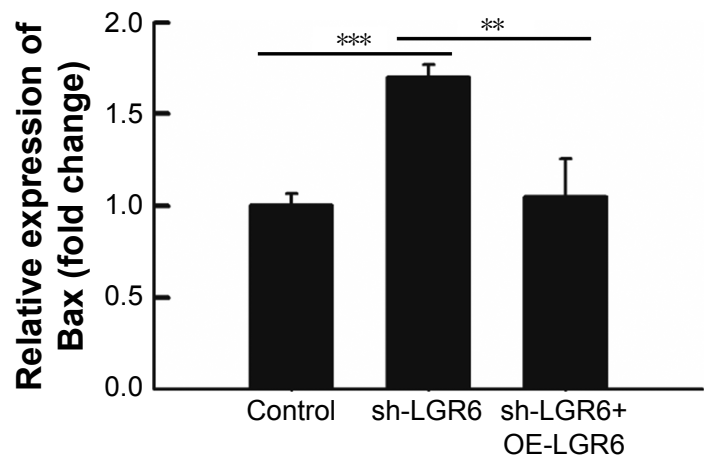

Figure 2 The effects of sh-LGR6 on the mRNA expression of GC cells.

Notes: The mRNA expression level was determined by RT-PCR and expressed as fold change relative to the vehicle group. Each column is shown as the mean of three separate experiments; bars, $S D$. $* * P<0.01$. $* * * P<0.001$.

Abbreviations: GC, gastric cancer; OE-LGR6, lentiviral expression vector of LGR6 gene; RT-PCR, reverse-transcription polymerase chain reaction; SD, standard deviation; sh-LGR6, lentiviral LGR6 shRNA.

\section{MGC803}
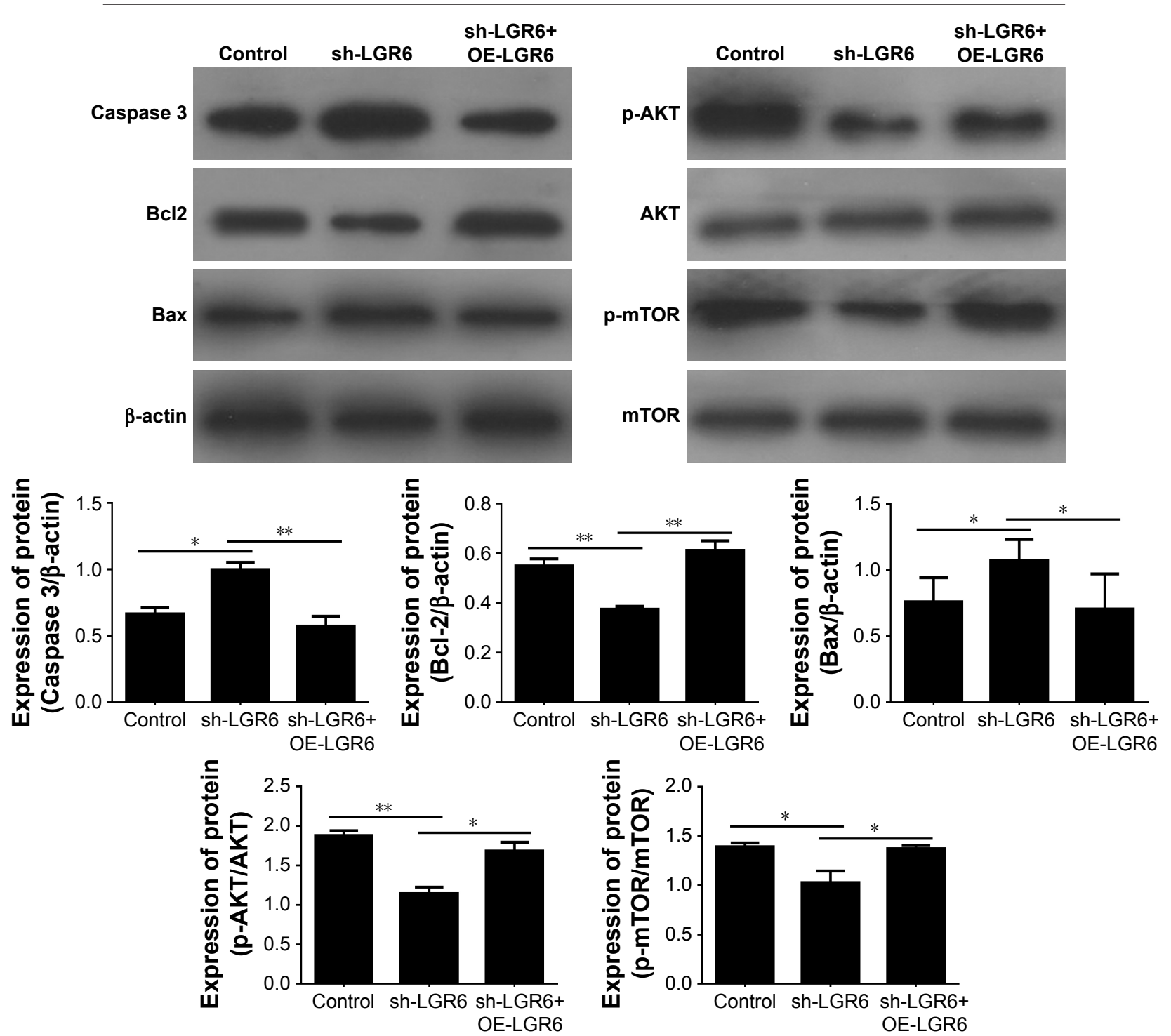

Figure 3 (Continued) 


\section{MKN45}
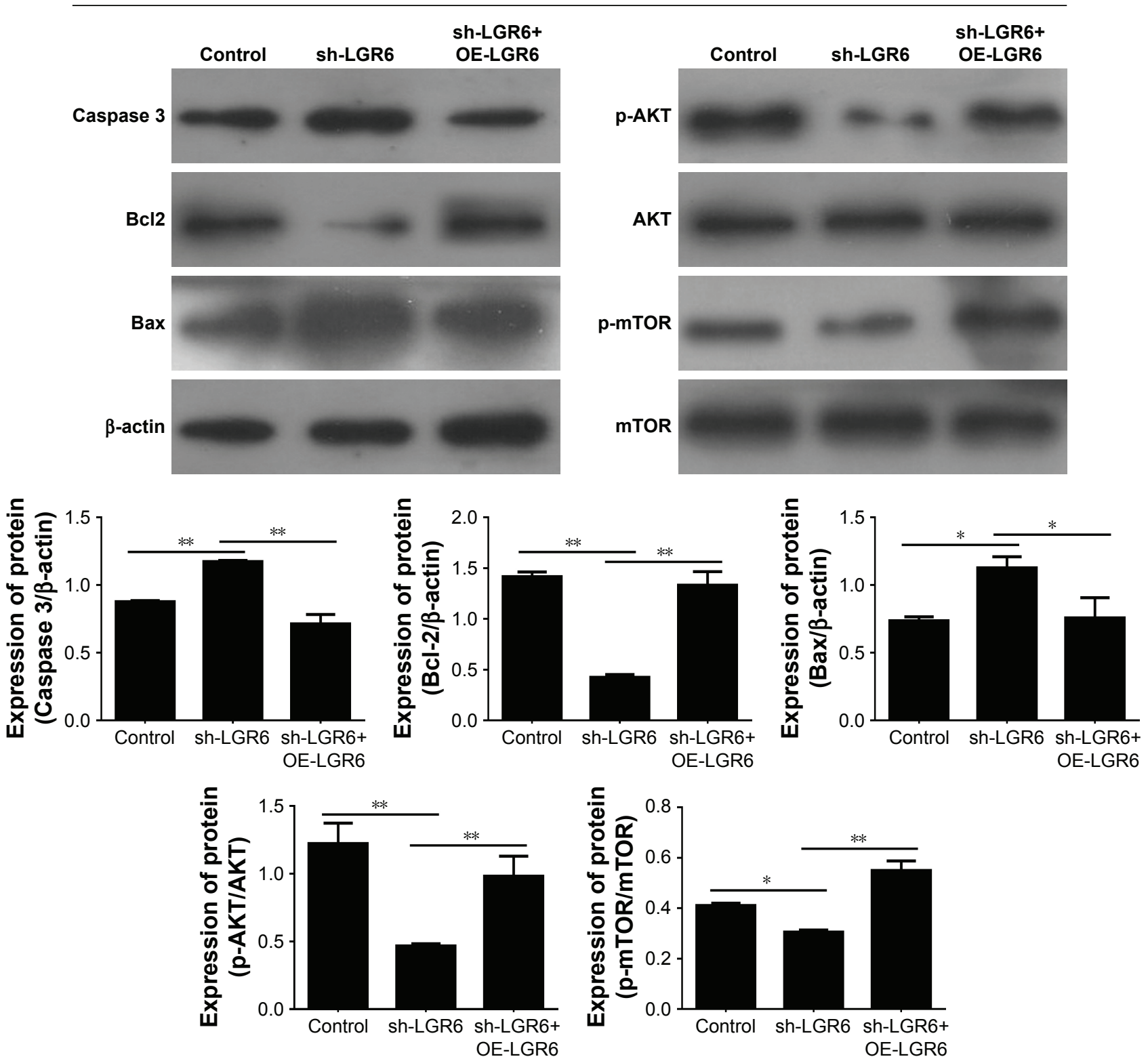

Figure 3 The effects of sh-LGR6 on the protein expression of GC cells.

Notes: Caspase 3, Bcl2, Bax, and AKT/mTOR signaling protein level in MGC803 and MKN45 cell lines treated with vehicle, sh-LGR6 or sh-LGR6+OE-LGR6 were detected by Western blot. Each column is shown as the mean of three separate experiments; bars, $S D$. $* P<0.05$. $* * P<0.01$.

Abbreviations: GC, gastric cancer; OE-LGR6, lentiviral expression vector of LGR6 gene; SD, standard deviation; sh-LGR6, lentiviral LGR6 shRNA.

PI3K/AKT/mTOR pathway, and overexpression of LGR6 promoted the activation.

\section{The roles of LGR6 in the proliferation and apoptosis of gastric cells}

The proliferation of GC cells was quantified by MTT assay. The result showed that LGR6 played an important role in the proliferation of GC cells. sh-LGR6 could inhibit the proliferation at 24, 48, 72, and $96 \mathrm{~h}$ after seeding (Figure 4). Given that LGR6 could regulate AKT/mTOR pathway, we detected the role of LGR6 in the apoptosis of GC cells by flow cytometry. We found that reduced LGR6 expression could significantly promote the apoptosis of gastric cells $(P<0.001$; Figure 4); LGR6 overexpression inhibited the apoptosis of GC cells $(P<0.001$; Figure 4$)$.

\section{LGR6 inhibits the invasion and migration of gastric cells}

The invasion and migration of gastric cells were investigated by wound healing assay and Transwell method, respectively. We found that LGR6 also plays an important role in the invasion and migration of GC cells. The results showed that 

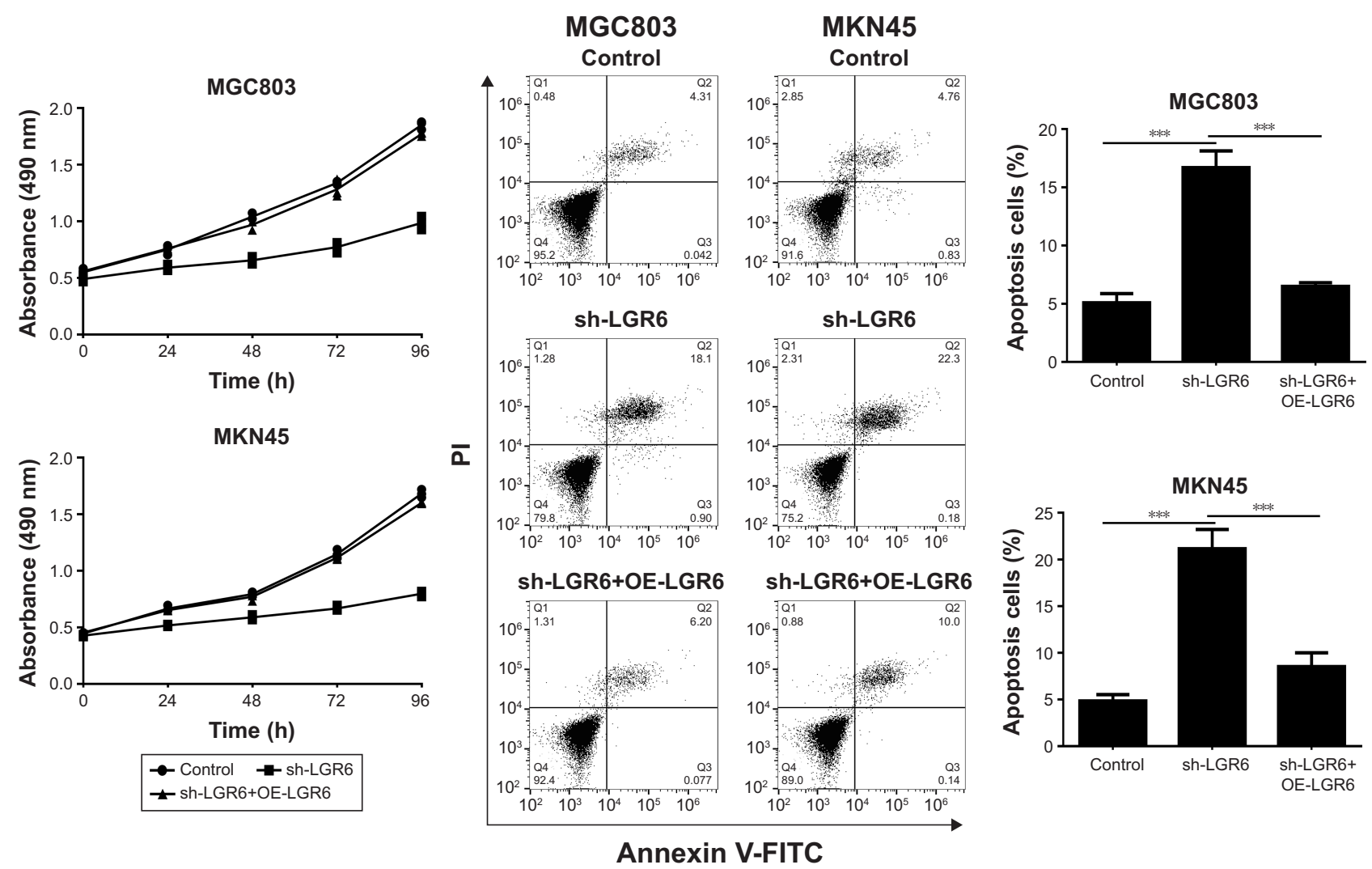

Figure 4 The effects of sh-LGR6 on proliferation and apoptosis of gastric cells.

Notes: The proliferation was evaluated by MTT assay. Each point is shown as the mean of three separate experiments. The apoptosis was evaluated by FACS. The Annexin V-FITC changed into Annexin V-PE, and PI changed into 7-AAD. Representative images are shown. Each column is shown as the mean of three separate experiments; bars, SD. *** $<0.00$ I. Abbreviations: FACS, fluorescence-activated cell sorter; OE-LGR6, lentiviral expression vector of LGR6 gene; SD, standard deviation; sh-LGR6, lentiviral LGR6 shRNA.

sh-LGR6 could significantly inhibit the invasion and migration of GC cells $(P<0.05$; Figure 5); LGR6 overexpression promoted the invasion and migration of GC cells $(P<0.05$; Figure 5).

\section{Discussion}

$\mathrm{PI} 3 \mathrm{~K} / \mathrm{AKT} / \mathrm{mTOR}$ pathway is an important intracellular signal transduction pathway to control the progression of tumor cells, including apoptosis, transcription, translation, metabolism, and angiogenesis, by influencing the activation state of many downstream effect molecules..$^{5,19-21}$ Therefore, we hypothesized that PI3K/AKT/mTOR pathway was involved in the tumor progression regulated by LGR6. This hypothesis has been confirmed by the present result which showed that phosphorylated AKT and mTOR could be downregulated by sh-LGR6.

The antiapoptotic effect of PI3K/AKT/mTOR pathway may be related to the following mechanisms: 1) Regulating the activity of Bcl-2 family members. The Bcl-2 protein family has been divided into two categories according to its function: one is Bcl-2 and Bcl-xL which inhibit apoptosis, and the other is proteins that promote apoptosis, such as
Bax, Bad, Bik, and Bid. Cell survival or apoptosis depends on the balance between these two categories. ${ }^{22}$ 2) Caspase-9 is involved in the initiation of apoptosis and Caspase- 3 is involved in the execution of apoptosis. Activated AKT can phosphorylate Caspase- 9 and Caspase- 3 to prevent Caspase- 9 and Caspase- 3 activation. ${ }^{23}$ In our result, the expression of proapoptotic proteins Bax and Caspase-3 were upregulated by sh-LGR6; the expression of antiapoptotic protein $\mathrm{Bcl} 2$ was downregulated by sh-LGR6. This indicated that LGR6 is an antiapoptosis protein which controls the apoptosis of GC cells through PI3K/AKT/mTOR pathway. Moreover, we performed functional experiments to figure out the regulation of LGR6 on the progression of GC cells. The results showed that LGR6 controlled not only the apoptosis but also the proliferation, migration, and invasion of GC cells. It might be due to the complicated function of PI3K/AKT/mTOR pathway on the progression of tumor cells. Of course, other pathways, such as Wnt/ $\beta$-catenin pathway, may also play an important role in this process.

As a member of the LGRs, LGR6 is most homologous to two other receptors, LGR4 and LGR5, with 50\% identity between each other at the amino acid level. ${ }^{24}$ Several studies 

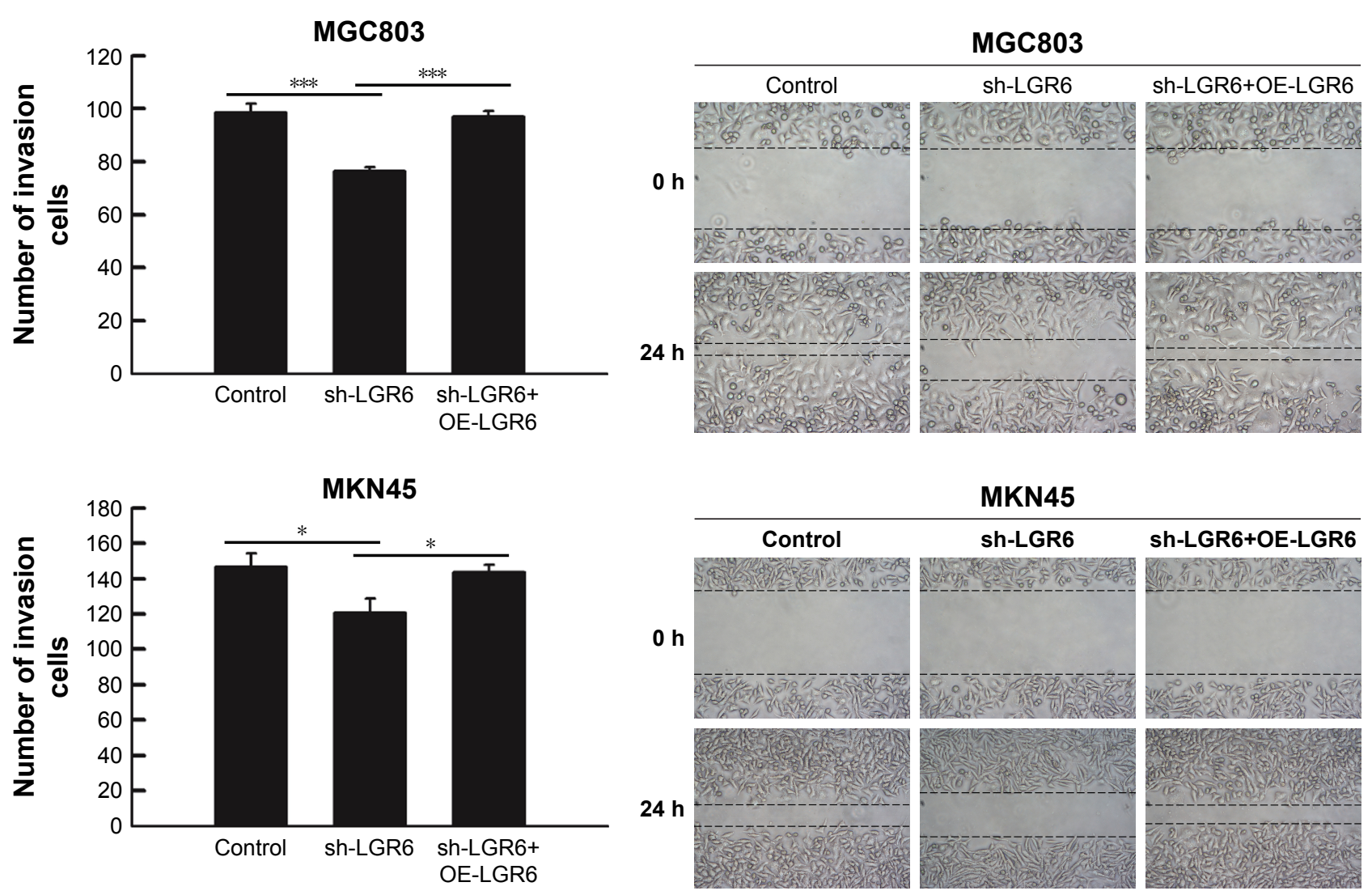

Figure 5 The effects of sh-LGR6 on invasion and migration of gastric cells.

Notes: The invasion was evaluated by Transwell assay. Each column is shown as the mean of three separate experiments; bars, SD. $* P<0.05$. $* * * P<0.00 I$. The migration was evaluated by wound healing assay. Representative images are shown. The magnification I00X.

Abbreviations: OE-LGR6, lentiviral expression vector of LGR6 gene; SD, standard deviation; sh-LGR6, lentiviral LGR6 shRNA.

have reported that LGR4 and LGR5 are associated with various types of cancer. LGR4 expression was shown to be increased in GC and is useful in diagnosis and prognostic evaluation of GC. ${ }^{25,26}$ For LGR5, earlier studies suggested that the expression of LGR5 is significantly higher in GC and LGR 5 overexpression significantly enhanced the growth, proliferation, migration, and drug resistance of GC cells..$^{27,28}$ At present, the study on the role of LGR6 in the progression of tumor cells is few. Gong et a ${ }^{13}$ reported that LGR6 could activate the $\mathrm{Wnt} / \beta$-catenin pathway to increase cell migration. $\mathrm{Wnt} / \beta$-catenin pathway is also involved in the function of LGR4 and LGR5..$^{29,30}$ This is the first study providing robust evidence that PI3K/AKT/mTOR pathway was involved in the function of LGR6. That may provide a new direction to research the functions of LGR4 and LGR5.

There were still some limitations in our study. First, we only conducted experiments in vitro. To get a more correct conclusion, we should conduct experiments in vivo. Second, this study investigated the roles of LGR6 overexpression in the progression of GC cells by comparing sh-LGR6 group with sh-LGR6+OE-LGR6 group. However, OE-LGR6 group may be needed to identify the role of LGR6 overexpression in wild-type GC cells. Third, in the functional experimental portion, an experimental group for PI3K/AKT/mTOR pathway inhibitor should be designed to study whether $\mathrm{PI} 3 \mathrm{~K} / \mathrm{AKT} / \mathrm{mTOR}$ pathway plays a major role in the function of LGR6.

\section{Conclusion}

Our findings indicated that LGR6 could promote the progression of GC through PI3K/AKT/mTOR pathway. That contributes to figuring out the molecular mechanisms of GC progression. In the future, LGR6 may become a new therapeutic target of GC. More experiments in vivo and clinical trials are still needed.

\section{Disclosure}

The authors report no conflicts of interest in this work.

\section{References}

1. Fock KM. Review article: the epidemiology and prevention of gastric cancer. Aliment Pharmacol Ther. 2014;40(3):250-260. 
2. Chen W, Zheng R, Baade PD, et al. Cancer statistics in China, 2015. CA Cancer J Clin. 2016;66(2):115-132.

3. Li SC, Lee CH, Hung CL, Wu JC, Chen JH. Surgical resection of metachronous hepatic metastases from gastric cancer improves long-term survival: a population-based study. PLoS One. 2017;12(7):e0182255.

4. Kang JM, Park S, Kim SJ, et al. KIAA1324 suppresses gastric cancer progression by inhibiting the oncoprotein GRP78. Cancer Res. 2015; 75(15):3087-3097.

5. Lang GD, Konda VJ. Early diagnosis and management of esophageal and gastric cancer. Minerva Gastroenterol Dietol. 2013;59(4): 357-376.

6. Barker N, Clevers H. Leucine-rich repeat-containing G-protein-coupled receptors as markers of adult stem cells. Gastroenterology. 2010; 138(5):1681-1696.

7. Snippert HJ, Haegebarth A, Kasper M, et al. Lgr6 marks stem cells in the hair follicle that generate all cell lineages of the skin. Science. 2010;327(5971):1385-1389.

8. Bonora M, Wieckowsk MR, Chinopoulos C, et al. Molecular mechanisms of cell death: central implication of ATP synthase in mitochondrial permeability transition. Oncogene. 2015;34(12):1608.

9. Ren W, Lewandowski BC, Watson J, et al. Single Lgr5- or Lgr6expressing taste stem/progenitor cells generate taste bud cells ex vivo Proc Natl Acad Sci U S A. 2014;111(46):16401-16406.

10. Leushacke M, Barker N. Lgr5 and Lgr6 as markers to study adult stem cell roles in self-renewal and cancer. Oncogene. 2012;31(25):3009-3022.

11. Guinot A, Oeztuerk-Winder F, Ventura JJ. miR-17-92/p38alpha dysregulation enhances wnt signaling and selects Lgr6+ cancer stem-like cells during lung adenocarcinoma progression. Cancer Res. 2016;76(13) 4012-4022.

12. Krejs GJ. Gastric cancer: epidemiology and risk factors. Digestive Dis. 2010;28(4-5):600-603.

13. Gong X, Carmon KS, Lin Q, Thomas A, Yi J, Liu Q. LGR6 is a high affinity receptor of R-spondins and potentially functions as a tumor suppressor. PLoS One. 2012;7(5):e37137.

14. Vogelstein B, Kinzler KW. Cancer genes and the pathways they control. Nature Med. 2004;10(8):789-799.

15. Chi J, Pierides C, Mitsidou A, Miltiadou A, Gerasimou P, Costeas P. cDNA synthesis for BCR-ABL1 detection at the MMR level: the importance of using the appropriate kit. Biol Proced Online. 2015;17(1):4.

16. Mahdavinezhad A, Yadegarazari R, Mousavi-Bahar SH, et al. Evaluation of zinc finger E-box binding homeobox 1 and transforming growth factor-beta2 expression in bladder cancer tissue in comparison with healthy adjacent tissue. Investig Clin Urol. 2017;58(2):140-145.
17. Schneider CA, Rasband WS, Eliceiri KW. NIH Image to ImageJ: 25 years of image analysis. Nat Methods. 2012;9(7):671-675.

18. Lei H, Xu Y, Guan R, et al. Effect of gyromagnetic fields on human prostatic adenocarcinoma cells. Onco Targets Ther. 2015;8:3489-3497.

19. Tian B, Zhao Y, Liang T, et al. Curcumin inhibits urothelial tumor development by suppressing IGF2 and IGF2-mediated PI3K/AKT/ mTOR signaling pathway. J Drug Target. 2017;25(7):626-636.

20. Mitra A, Raychaudhuri SK, Raychaudhuri SP. IL-22 induced cell proliferation is regulated by $\mathrm{PI} 3 \mathrm{~K} / \mathrm{Akt} / \mathrm{mTOR}$ signaling cascade. Cytokine. 2012;60(1):38-42.

21. Papadimitrakopoulou V. Development of PI3K/AKT/mTOR pathway inhibitors and their application in personalized therapy for non-smallcell lung cancer. J Thorac Oncol. 2012;7(8):1315-1326.

22. Henshall DC, Araki T, Schindler CK, et al. Activation of Bcl-2associated death protein and counter-response of Akt within cell populations during seizure-induced neuronal death. J Neurosci. 2002; 22(19):8458-8465.

23. Altieri DC. The molecular basis and potential role of survivin in cancer diagnosis and therapy. Trends Mol Med. 2001;7(12):542-547.

24. Hsu SY, Kudo M, Chen T, et al. The three subfamilies of leucine-rich repeat-containing $\mathrm{G}$ protein-coupled receptors (LGR): identification of LGR6 and LGR7 and the signaling mechanism for LGR7. Mol Endocrinol. 2000;14(8):1257-1271.

25. Xiao Y, Chen L. [Research progress of Lgr4 in gastrointestinal carcinomas]. Zhonghua wei chang wai ke za zhi. 2015;18(11): $1179-1181$

26. Wang Y, Dong J, Li D, et al. Lgr4 regulates mammary gland development and stem cell activity through the pluripotency transcription factor Sox2. Stem Cells. 2013;31(9):1921-1931.

27. Wang B, Chen Q, Cao Y, et al. LGR5 is a gastric cancer stem cell marker associated with stemness and the EMT signature genes NANOG, NANOGP8, PRRX1, TWIST1, and BMI1. PLoS One. 2016; 11(12): 0168904.

28. Li XB, Yang G, Zhu L, et al. Gastric Lgr5(+) stem cells are the cellular origin of invasive intestinal-type gastric cancer in mice. Cell Res. 2016;26(7):838-849.

29. de Sousa EMF, Colak S, Buikhuisen J, et al. Methylation of cancer-stemcell-associated Wnt target genes predicts poor prognosis in colorectal cancer patients. Cell Stem Cell. 2011;9(5):476-485.

30. Walker F, Zhang HH, Odorizzi A, Burgess AW. LGR5 is a negative regulator of tumourigenicity, antagonizes Wnt signalling and regulates cell adhesion in colorectal cancer cell lines. PLoS One. 2011;6(7): e22733.
OncoTargets and Therapy

\section{Publish your work in this journal}

OncoTargets and Therapy is an international, peer-reviewed, open access journal focusing on the pathological basis of all cancers, potential targets for therapy and treatment protocols employed to improve the management of cancer patients. The journal also focuses on the impact of management programs and new therapeutic agents and protocols on

\section{Dovepress}

patient perspectives such as quality of life, adherence and satisfaction. The manuscript management system is completely online and includes a very quick and fair peer-review system, which is all easy to use. Visit http://www.dovepress.com/testimonials.php to read real quotes from published authors. 